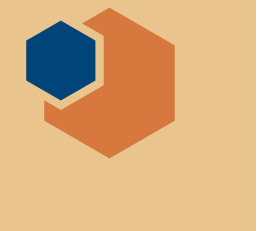

\title{
Can extreme materials usher in a nuclear renaissance?
}

Thirty-five years to the date following the worst technological disaster in history, still-glowing embers entombed inside Chernobyl's reactor recently made headlines. Yet with the specter of global climate change looming ever greater, nuclear power could remain a viable, if controversial, part of the solution along with other renewable energy sources.

\section{$\mathrm{T}$}

2019 Chernobyl television miniseries, with its harrowing "click-click-click" Geiger counter beat, reverberated many of the fears that society holds about nuclear power. The show's mostly accurate depiction of the catastrophic 1986 event, and the confluence of flawed engineering design and operational mismanagement that converted a civilian source of electrical power into the worst techno-ecological disaster of human history, reinvigorated debate about the role of this 60-year-old energy source. The Chernobyl explosion stands front and center in a line of several infamous nuclear power catastrophes, including Fukushima and Three Mile Island. Nevertheless, according to the US Energy Information Administration, the United States relies on 94 nuclear reactors at 56 power plants to supply 96.6 GW of electrical power every day to its homes and businesses. These sites are present in 28 states across the country, along with numerous additional facilities that process fuel and test new reactor concepts, as well as mobile military-operated nuclear reactors onboard submarines and aircraft carriers.

US nuclear power plants produced 790 million megawatt-hours (MWh) last year and accounted for one-fifth of energy generation nationwide. At the same time, nuclear power is expensive: a MWh from each one costs between USD $\$ 112$ and $\$ 183$, whereas natural gas $(40 \%$ of total US electricity generation) costs USD\$42-\$78 per MWh. While projections estimate a $50 \%$ worldwide energy consumption increase from 2015 levels by year 2040, nuclear power's contribution to the corresponding supply remains uncertain. The International Energy Agency's 2021 Global Energy Review noted curtailed nuclear power operations in the European Union $(-11 \%)$, Japan $(-33 \%)$, and the United States $(-2 \%)$. The 2011 Fukushima incident, along with cost concerns and pandemic-driven dips in energy demand, contributed to these drawdowns. Nevertheless, research into novel reactor and fuel designs may transform the value of nuclear power and, ultimately, its role in

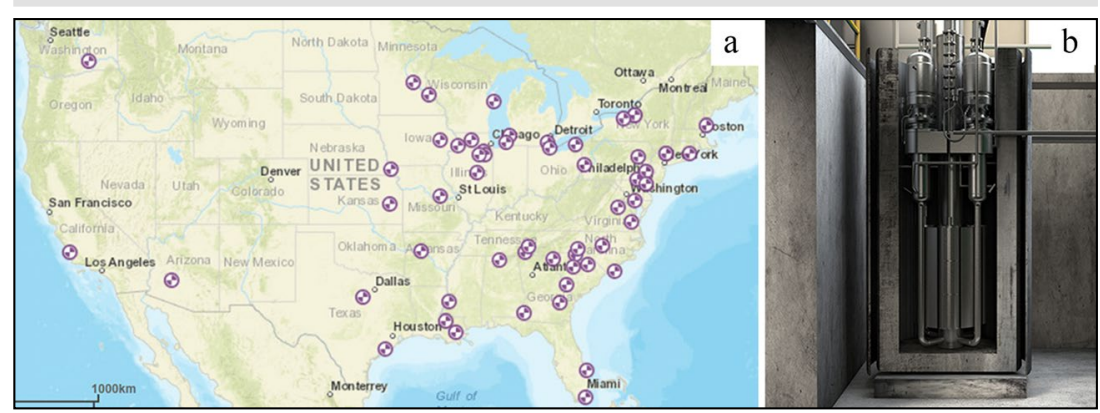

(a) Location of US nuclear power plants. Source: US Energy Information Administration, 2020. (b) Microreactor Applications Research Validation and EvaLuation (MARVEL) liquidmetal cooled 100-kW microreactor. Source: Idaho National Laboratory/US Department of Energy. worldwide energy supply and the battle against climate change.

According to a 2019 Congressional Research Services (CRS) report, Advanced Nuclear Reactors: Technology Overview and Current Issues, all nuclear power stations in the United States use light water reactors. They require fuel rods that contain fuel pellets of non-fissile uranium-238 isotope and a small weight fraction ( $5 \%$ or less) of the fissile uranium-235. During the reactors' operation, fuel rods are inserted into the reactor, and emitted neutrons from fissile atoms bombard the remaining uranium atoms to propagate nuclear fission. Regular water slows down ("moderates") the neutrons to speeds that allow this process to propagate at a steady, sustainable rate. The reactor transfers released heat into water and uses it to revolve turbines that generate electrical power. According to Yutai Katoh, a research scientist at Oak Ridge National Laboratory who leads efforts to develop novel materials for nuclear fuel rods, construction of the reactor itself is the costliest segment of nuclear power. The CRS report further notes that complex regulations, shifting market demands, and technological challenges contribute to cost overruns and expensive delays during construction of these reactors.

As Katoh told MRS Bulletin, the stateof-the-art reactor design only allows use of less than $1 \%$ of the total energy that the fuel pellets can potentially generate. Whereas a drastic increase in energy extraction would require a novel reactor design, increased uranium-235 burn-up and higher operating temperatures can 
boost efficiencies. However, existing fuel cladding, which uses zirconium alloys, does not offer the temperature stability and reliability to allow either capability. "An advanced high temperature, accident-tolerant fuel cladding coupled with advanced high burn-up fuel pellets is the key for nuclear energy to claim leadership in baseload electricity in the new era of carbon-free energy," Katoh said. Materials solutions that may improve cost-competitiveness include various nanostructured iron-chromiumaluminum-based alloys and ceramic matrix composites. New characterization techniques provide researchers better views into radiation effects on these fuel cladding solutions. Furthermore, recent progress in advanced manufacturing capabilities, such as 3D printing, may allow customized designs for these difficult-to-manufacture multimaterial integrations and allow less expensive and more efficient fuels to safely operate during their expected lifetime - which often exceeds 18 months.

Development of next-generation fission reactors typically falls into one of two categories. The first aims to scale down the reactors from their conventional $1000-\mathrm{MW}$ output to $300 \mathrm{MW}$ or less. A second approach changes the overall reactor design and operates at high temperatures and pressures for the fuel to deliver higher efficiencies. As the flagship government agency that oversees nuclear technology in the United States, the Department of Energy (DOE) carries out most of the programs that develop new nuclear reactor concepts and fuels. Through the National Reactor Innovation Center, its Advanced Reactor Demonstration Program has allocated USD\$160 million for public-private partnerships that aim to accelerate development and demonstration of novel reactors. The Versatile Test Reactor, which is slated to come online in 2026, is a one-of-a-kind user facility that will enable essential testing of new power-generating designs.

The modular reactor approach leverages scalable advanced manufacturing technologies that can rapidly and inexpensively produce parts for this miniaturized technology and strategically deploy a greater number of powerproducing units on the grid. The DOE recently announced its Microreactor Applications Research Validation and EvaLuation Project (MARVEL), which will be built at the Idaho National Laboratory to deliver $100 \mathrm{~kW}$ of fission power in three years' time. This prototype is expected to take advantage of highly enriched nuclear fuel. As noted in the CRS report, GE Hitachi, Westinghouse, $\mathrm{NuTec}$, and other private companies are also developing microreactors. Fast fabrication, possibly aided by advances in additive manufacturing and machine-learning-driven design, is expected to offset costs from construction delays of large conventional reactors. However, as a 2013 Union of Concerned Scientists report noted, this approach eschews the economies of scale that benefit larger power plants and may not deliver the anticipated cost savings.

While conventional light water reactors operate at $330^{\circ} \mathrm{C}$, next-generation power plant concepts require temperatures as high as $1000^{\circ} \mathrm{C}$. As highlighted in the CRS report and a 2015 RAND Corporation Perspective, "Overcoming Obstacles to Advanced Reactor Technologies," a variety of reactor concepts are in development. Many use fast neutrons to accelerate atom splitting, while some use liquid sodium or gas to transfer heat from the fuel to the turbine. New fuel concepts, which contain highly enriched fuel into billiardsized balls rather than cylindrical rods, are necessary for these reactor designs. Naturally, these designs require exceptionally durable materials that can withstand the extremely hot, high-pressure, and chemically corrosive environment while sustaining intense radiation bombardment - without as much as a hint of any deformation or failure.

The DOE is not the only federal entity that is looking to capitalize on potential benefits of new reactor designs. Ever since the first space launches of the 1950s, multiple efforts have aimed to capitalize on energy densities of nuclear power for space propulsion. While these efforts have, to date, remained within the realm of science fiction, several new programs have breathed new life into this idea. In April 2021, the US Defense Advanced Research Projects Agency announced three performers for its Demonstration Rocket for Agile Cislunar Operations (DRACO) program. According to the announcement, the program's goal is to demonstrate nuclear thermal propulsion for a spacecraft in low-Earth orbit by 2025 , and, eventually, enable greater thrust capabilities for travel between the Earth and Moon. NASA's recently concluded Kilopower project similarly assessed nuclear fission-driven propulsion for its spacecraft to develop highspeed engines in place of ion thrusters. The latter require plutonium isotopes, which are becoming scarcer and more expensive. Although both programs remain in the exploratory development stages, their successes could transcend the limits of current chemical rocket technologies and revolutionize the entire concept of space travel. These efforts align with a January 2021 Executive Order on "Promoting Small Modular Reactors for National Defense and Space Exploration" that had been signed by the outgoing US President.

In addition to reactor design, the US nuclear industry must confront challenges at both beginning and ends of the fuel cycle in its efforts to decrease costs and ensure safety.

A 2019 CRS report, The Front End of the Nuclear Fuel Cycle: Current Issues, notes that the US imports $93 \%$ of the uranium that it uses in its nuclear reactors. Subsequently, this supply chain is vulnerable to commodity price fluctuations, political actions such as tariffs and supply quotas, and shutdowns akin to those that had rippled through many industries during the COVID19 pandemic. The United States can no longer benefit from the "Megatons to Megawatts" program, which, from 1993 until 2013, inexpensively supplied decommissioned warheads from the 
former Soviet Union for reprocessing into commercial reactor fuel. Under the program, the government-formed United States Enrichment Corporation imported 500 tons of bomb-grade uranium and converted them into 14,000 tons of nuclear fuel that accounted for $10 \%$ of the country's supply-over USD\$12 billion worth of fuel for a cost of USD\$8 billion. A 2019 US Department of Commerce report concluded that the trade imbalance threatened the national security of the United States. A multiagency Nuclear Fuel Working Group (led by the DOE) formed in response. In April 2020, it published a report that highlighted domestic uranium mining and advanced fuel reprocessing $R \& D$ as critical routes that can help secure the domestic supply of this energy source.

Nuclear power plants hold an advantage over fossil-fuel-burning counterparts: the fuel of the former does not offgas as $\mathrm{CO}_{2}$ to the atmosphere. However, spent nuclear fuel from commercial power plants is highly radioactive and must be appropriately managed. The most recent assessment made by the US Government Accountability Office had estimated the total volume of this waste to be 80,000 metric tons. The Nuclear Regulatory Commission, a US government agency that provides much of the oversight on nuclear power safety, permits power plants to store spent and cooled-down fuel in sealed containers. These "dry cask" stores are situated directly on the sites of the power plants, and, at this time, function as primary life-cycle end points for spent fuel rods.

A 2020 CRS report, Civilian Nuclear Waste Disposal, notes that the 1982 Nuclear Waste Policy Act, which had designated a deep geological repository as the permanent storage site for this waste, has yet to establish and fully operate such a site. The Yucca Mountain repository in Nevada has faced significant pushback and is currently inoperable. The Waste Isolation Pilot Plant in New Mexico is the only centralized location that can permanently hold transuranic waste; however, its use is solely for defense applications and it cannot hold commercial nuclear fuel. Dylan Rittman, a former Optical Society/Materials Research Society Congressional Fellow and a subject matter expert on nuclear waste, highlighted the challenges of designing containment materials and storage facilities that withstand radiation damage, environmental effects, and, ultimately, changing geology. "The materials science community has done great work designing materials that can safely contain nuclear waste for thousands to tens of thousands of years. The challenge with Yucca Mountain, or any long-term commercial nuclear waste repository in the US," he told MRS Bulletin, "is the regulatory standard of a quantitative risk assessment out to 1 million years. This standard exists nowhere else in the world, and confidently projecting behavior out to these time scales requires a deep understanding of the interplay between materials science and geology."

Nuclear power has received renewed attention in the US Congress. The Energy Act of 2020, which was signed into law at the end of 2020 as a component of the omnibus spending package, allocated funding for research, development, and commercialization of next-generation nuclear reactors. Senators from both political parties recently re-introduced the Financing Our Energy Future Act and discussion drafts of the Energy Sector Innovation Credit Act. These legislative items, respectively, aim to encourage master limited partnerships (a publicly traded entity taxed as a partnership) and tax incentives for development of novel nuclear power generation technology. The Republican minority in the US House of Representatives recently unveiled a package of six bills that aim to spur nuclear power development in a targeted effort to combat greenhouse gas emissions and climate change. A hearing held before the US Senate Committee on Energy and Natural Resources in March 2021 examined a series of recent developments in the nuclear energy sector. Witness testimony from five industry experts focused on the role of nuclear power in clean energy and the need for innovation that can help bring about less expensive, more efficient, and safer reactor technologies.

Notwithstanding these efforts, growth of nuclear energy has effectively stalled in the United States. According to a 2020 CRS report, Nuclear Energy: Overview of Congressional Issues, only two new power stations are currently under construction. Even more are reaching the end of their life and are shutting down; most recently, New York's Indian Point generating station ceased operations in April 2021. In addition to costs and regulatory burdens, safety concerns nosedive public support for additional nuclear power. While Chernobyl, Fukushima, and the Three Mile Island incidents remain well known, a 2015 MIT Technology Review article, which had compiled a list of 102 accidents within the nuclear industry, calculated a $50 \%$ chance of another Chernobyl-scale catastrophe occurring before the year 2050. Moreover, while new nuclear designs may offer greater efficiencies, they require highly enriched uranium and plutonium, which also raise dual-use and proliferation concerns. Nuclear power remains controversial, but it also persists as an important energy-dense power-generating source of electricity.

Boris Dyatkin

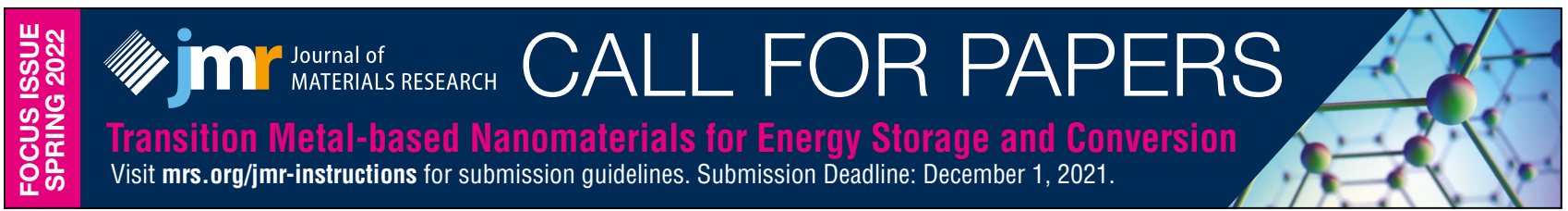

